\title{
glm2: Fitting Generalized Linear Models with Convergence Problems
}

\author{
by Ian C. Marschner
}

\begin{abstract}
The R function $\mathrm{glm}$ uses step-halving to deal with certain types of convergence problems when using iteratively reweighted least squares to fit a generalized linear model. This works well in some circumstances but non-convergence remains a possibility, particularly with a nonstandard link function. In some cases this is because step-halving is never invoked, despite a lack of convergence. In other cases step-halving is invoked but is unable to induce convergence. One remedy is to impose a stricter form of stephalving than is currently available in $\mathrm{glm}$, so that the deviance is forced to decrease in every iteration. This has been implemented in the glm2 function available in the glm2 package. Aside from a modified computational algorithm, glm2 operates in exactly the same way as glm and provides improved convergence properties. These improvements are illustrated here with an identity link Poisson model, but are also relevant in other contexts.
\end{abstract}

It is not too uncommon for iteratively reweighted least squares (IRLS) to exhibit convergence problems when fitting a generalized linear model (GLM). Such problems tend to be most common when using a nonstandard link function, such as a log link binomial model or an identity link Poisson model. Consequently, most commonly used statistical software has the provision to invoke various modifications of IRLS if non-convergence occurs.

In the stats package of R, IRLS is implemented in the $g l \mathrm{~m}$ function via its workhorse routine glm. fit. This routine deals with specific types of convergence problems by switching to step-halving if iterates display certain undesirable properties. That is, if a full Fisher scoring step of IRLS will lead to either an infinite deviance or predicted values that are invalid for the model being fitted, then the increment in parameter estimates is repeatedly halved until the updated estimates no longer exhibit these features. This is achieved through repeated application of the call

start $<-($ start + coefold $) / 2$

where coefold and start contain estimates from the previous and current iterations, respectively.

Although this approach works well in some contexts, it can be prone to fail in others. In particular, although the step-halving process in $\mathrm{glm}$. fit will throw an errant iterative sequence back into the desired region, the sequence may repeatedly try to escape that region and never converge. Furthermore, it is even possible for the IRLS iterative sequence to be such that step-halving is never invoked in glm. fit, yet the sequence does not converge. Such behavior is typically accompanied by a deviance sequence that increases in one or more of the iterations. This suggests a modification to $\mathrm{glm}$. fit which has been implemented in the glm2 package (Marschner, 2011).

As motivation for the proposed modification, we begin by discussing the potential for non-convergence using some numerical examples of the above types of behavior. The glm2 package is then discussed, which consists of a main function $\mathrm{glm} 2$ and a workhorse routine $\mathrm{glm}$. fit2. These are modified versions of $\mathrm{glm}$ and glm.fit, in which step-halving is used to force the deviance to decrease from one iteration to the next. It is shown that this modification provides improved convergence behavior.

\section{Non-convergence}

We start by providing illustrations of the two types of non-convergence alluded to above. Specifically, we will consider situations in which standard IRLS does not converge, and for which either: (i) the stephalving in glm.fit is invoked but cannot induce convergence; or (ii) the step-halving in glm.fit is never invoked despite the non-convergence of IRLS. These two types of behavior will be illustrated using an identity link Poisson regression model, which can be prone to convergence problems as the link function does not automatically respect the non-negativity of the Poisson means. This context is useful for studying the convergence properties of algorithms based on IRLS, because for this particular GLM a reliable alternative algorithm exists which is not based on IRLS, as described by Marschner (2010). While we focus on the identity link Poisson model here, the same behavior can occur in other models and the documentation for the glm2 package includes a log link binomial example.

In Table 3.2 of Agresti (2007) a data set is presented that is amenable to analysis using a linear Poisson model. The data set consists of a count response variable $y_{i}, i=1, \ldots, 173$, called Sa in Agresti (2007), with observed values ranging from 0 through 15 and a mean of 2.9. Also presented are a number of potential covariates. Here we define three covariates, $x_{i 1}, x_{i 2}$ and $x_{i 3}, i=1, \ldots, 173$, similarly to Marschner (2010). The first two of these covariates, $x_{i 1}$ and $x_{i 2}$, are defined by dichotomizing the covariates called $C$ and $S$ in Agresti (2007), such that $\left\{x_{i 1}\right\}=1\{C>3\}$ and $\left\{x_{i 2}\right\}=1\{\mathrm{~S}<3\}$. This yields two $0 / 1$ binary covariates with means 0.4 and 0.3 , respectively. The third 
covariate, $x_{i 3}$, is a continuous covariate called $\mathrm{W}$ in Agresti (2007), which is shifted here by subtracting the smallest value, so that it ranges from 0 through 12.5 with a mean of 5.3 .

Assuming $y_{i}$ is an observation from a Poisson distribution with mean $\mu_{i}$, the identity link model is

$$
\mu_{i}=\alpha_{0}+\alpha_{1} x_{i 1}+\alpha_{2} x_{i 2}+\alpha_{3} x_{i 3}
$$

with parameter vector $\theta=\left(\alpha_{0}, \alpha_{1}, \alpha_{2}, \alpha_{3}\right)$. This model can be fitted in $\mathrm{R}$ by first defining $\mathrm{a}$ response vector $\mathrm{y}$ with $\mathrm{y}[\mathrm{i}]=y_{i}$, and corresponding covariate vectors $\mathrm{x} 1, \mathrm{x} 2$ and $\mathrm{x} 3$ with $\mathrm{c}(\mathrm{x} 1[i], \mathrm{x} 2[i], \mathrm{x} 3[i])=\left(x_{i 1}, x_{i 2}, x_{i 3}\right)$, along with a data frame poisdata through the assignment poisdata <- data.frame $(y, x 1, x 2, x 3)$. The call to fit the model is then

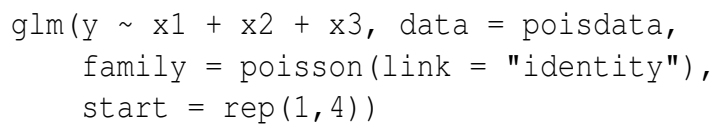

which includes an initial estimate through the start argument because the default choice is invalid for this model. Below we also use the control argument to monitor the iterative behavior.

As discussed later in the paper, the above call produces satisfactory convergence for these data; however, a bootstrap analysis using the same call leads to quite severe numerical instability. Using the sample function, bootstrap replications were generated by sampling with replacement from the original data, after which $g \mathrm{~lm}$ was applied to each replication. This process readily produced replications for which $\mathrm{glm}$ failed, and a collection of 100 such replications was generated in which non-convergence occurred. Two of these replications are discussed as examples in this section, while the full collection of 100 replications is discussed further in the next section.

Figure 1 displays the lack of convergence using $\mathrm{glm}$ for the two illustrative replications. Increasing the maximum number of iterations does not alleviate the problem. Also plotted in Figure 1 is the deviance achieved by the maximum likelihood estimate (MLE), calculated using the non-IRLS linear Poisson method of Marschner (2010). For Figure 1(a) this minimum possible deviance is 604.0 with an MLE of $\hat{\theta}=(-0.095,-0.385,0.618,0.530)$, while for Figure 1 (b) the minimum possible deviance is 656.3 with an MLE of $\hat{\theta}=(0.997,-1.344,-0.169,0.524)$. Inspection of the score functions reveals both MLEs are stationary and in the interior of the parameter space.

These two examples illustrate the two scenarios of non-convergence described at the beginning of this section. In Figure 1(a), step-halving was invoked in 28 of the 100 iterations, showing that $\mathrm{glm}$ can fail to converge even with step-halving. In Figure 1(b) stephalving was not invoked, showing that glm can fail to converge without ever making use of step-halving. The latter example is indicative of a potential problem with Newton-type algorithms, which can have a so-called attracting periodic cycle. In this case IRLS is attracted to a cycle of two iterate values, with deviances of 673.2 and 691.1, and then subsequently oscillates between those values.

Although these results use a specific starting value, the non-convergence cannot be remedied with better initial estimates. This is illustrated in Figure 1(a), where the iterates get very close to the optimal value. Only when the initial estimate is identical to the MLE does glm converge (in one iteration).

(a)

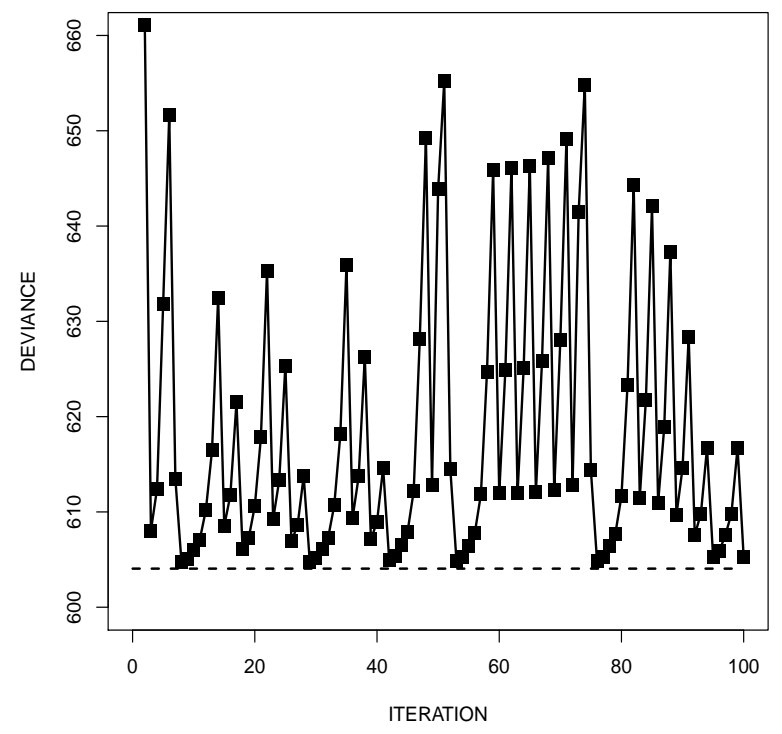

(b)

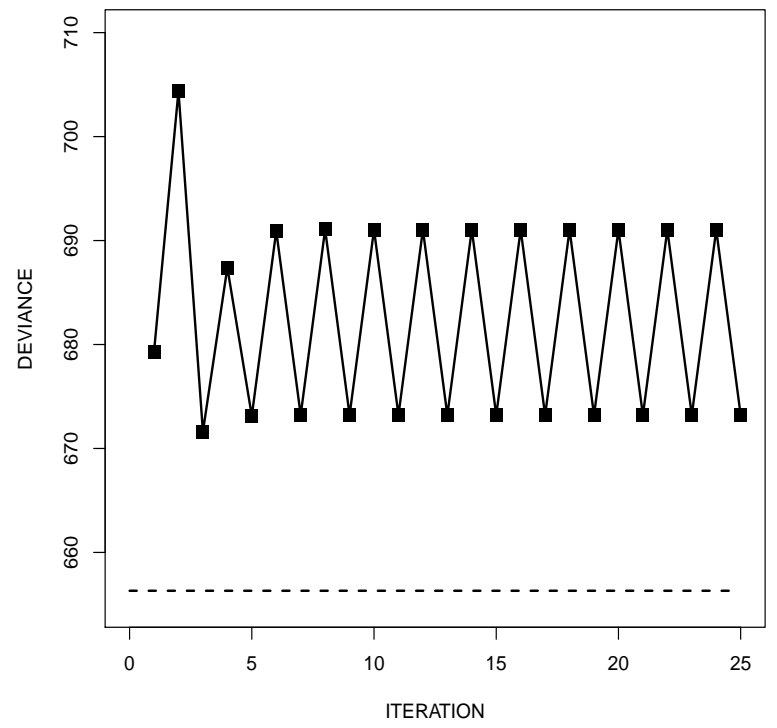

Figure 1: Examples of non-convergence in glm. Dashed lines denote the optimal deviance.

\section{The glm2 package}

An important feature of Figure 1 is that the iterative sequences display instances of increasing deviance 
from one iteration to the next. It is well known that step-halving can be used in Newton-type algorithms to force the objective function to improve monotonically (Lange, 2010, p. 251). This approach is used to improve the convergence properties of IRLS in other standard statistical software. Here we discuss its implementation in the glm2 function available within the glm 2 package, and the associated improvements in convergence properties.

The source code for glm. fit has two step-halving blocks called inner loops 1 and 2 . These occur within the main loop immediately prior to the test of convergence, and they use step-halving to rectify a divergent deviance and invalid predicted values, respectively. The main change implemented in the glm 2 package is that the modified routine glm. fit 2 has a further step-halving block, called inner loop 3, which tests whether the deviance is lower than in the previous iteration. If not, step-halving is invoked until the deviance is lowered, leading to an iterative sequence that monotonically decreases the deviance.

Convergence in glm.fit occurs if abs(rel) < control\$epsilon where rel is the relative change $(\mathrm{dev}-\mathrm{devold}) /(0.1+\mathrm{abs}(\mathrm{dev}))$ from the current deviance devold to the updated deviance dev, and control\$epsilon is a positive tolerance. In glm. fit2 the same convergence test is used, and if it is not satisfied because rel <= -control\$epsilon, then iterations proceed as in glm. fit. However, if convergence is not satisfied because rel >= control\$epsilon, then inner loop 3 invokes step-halving until rel <= -control\$epsilon. Thus, as well as decreasing the deviance, the step-halving takes $r e l$ from one side of the convergence region to the other, therefore never causing false convergence.

The glm2 function is essentially identical to $\mathrm{glm}$, except the default fitting method is glm. fit2. This allows glm2 to be called with the same calling sequence as glm. Alternatively, in version 2.12.1 and later, it should be possible to achieve the same effect by passing glm.fit2 to glm through the method argument, instead of the default method $\mathrm{glm}$. fit. Indeed, existing scripts calling $g \mathrm{~lm}$ should work unchanged with the new fitting function, after first executing

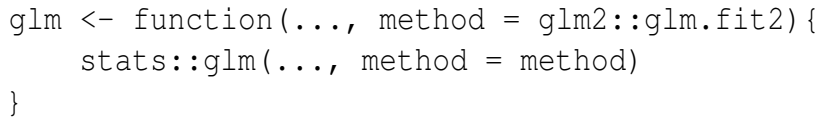

which makes glm.fit2 the default fitting method when $g l m$ is called.

In the previous section we discussed a data set for which 100 bootstrap replications were generated where glm failed to converge. When glm2 was used, convergence was achieved for all 100 replications with an initial value of 1 for each of the four parameters. This included the two illustrative examples plotted in Figure 1, which both converged to the MLEs produced by the non-IRLS method of Marschner (2010).
This convergence is displayed in Figure 2, together with the path that $g l m$ would take.

(a)

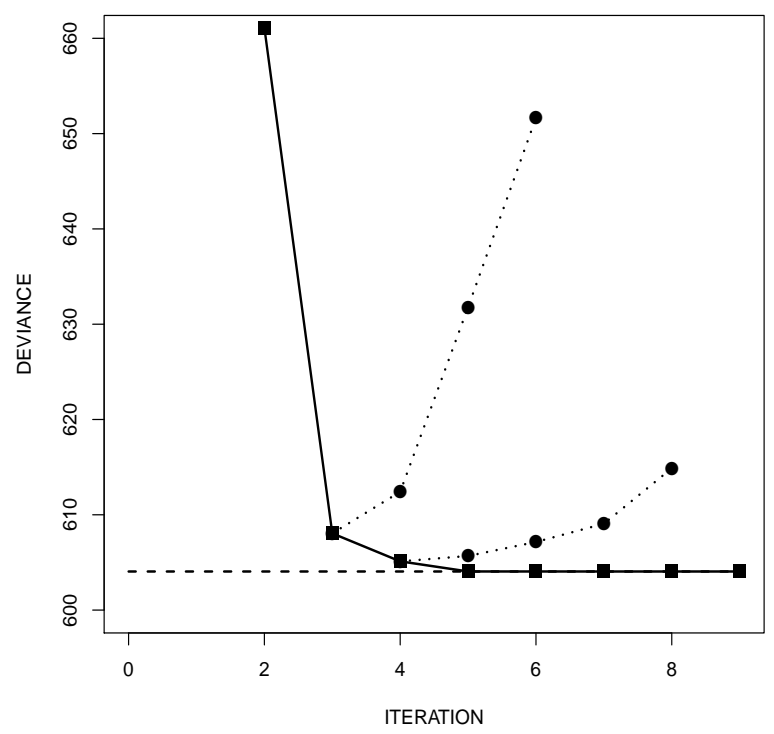

(b)

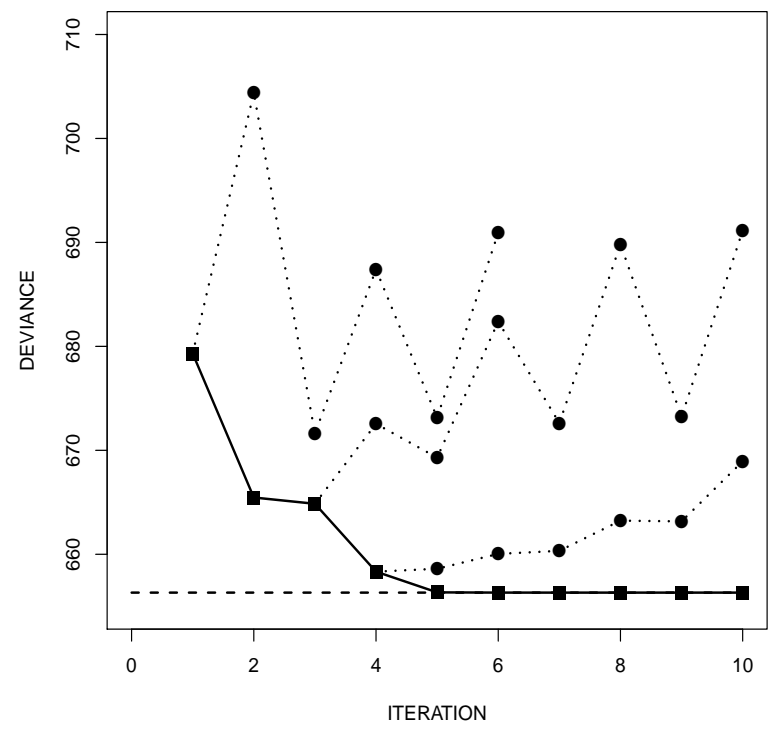

Figure 2: Convergence of glm2 (squares) for the examples presented in Figure 1. Circles denote the path that glm would take.

Using glm2, Figure 3 provides medians and interquartile ranges of the estimates from the 100 replications that did not converge in $\mathrm{glm}$. Also shown is the same information for 100 replications where $\mathrm{glm}$ did converge. In each case the two sets of estimates are relatively consistent. This would seem to suggest that estimates from the replications in which glm failed to converge are not particularly unusual compared to those for which it did converge. In particular, as demonstrated in Figure 1, non-convergence of $\mathrm{glm}$ can occur even when the MLE is a stationary point in the interior of the parameter space. 
As well as the functions glm2 and glm.fit2, the glm 2 package provides data sets and example code illustrating the usefulness of the proposed change. This allows reproduction of the behavior described here for the identity link Poisson model, and also provides an example for the log link binomial model.

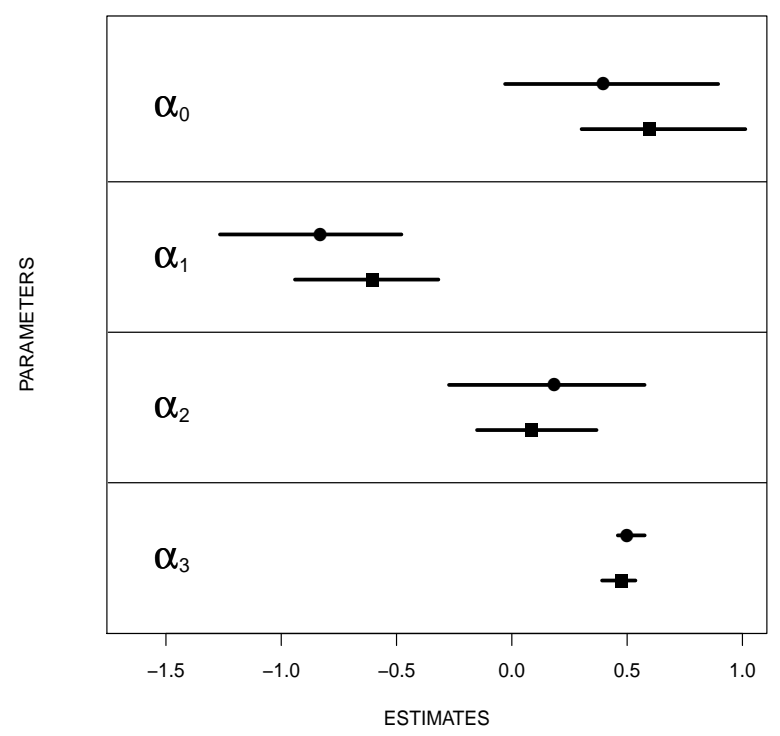

Figure 3: Medians and interquartile ranges of estimates in 100 replications that converged (squares) and 100 replications that did not converge (circles) in $\mathrm{glm}$. The latter were calculated using $\mathrm{g} l \mathrm{~m} 2$.

\section{Discussion}

This paper describes a modification that improves the convergence properties of $\mathrm{glm}$, and which is available in the glm2 function in the glm2 package. Following the recommendation of the editors, the modification has been presented as a separate package rather than as a code snippet. This has the advantage of facilitating thorough testing of the proposed change, and also allows some example analyses to be made available through the package. These examples are promising, but additional testing would be required before considering a change to $\mathrm{glm}$ and its ancillaries in the standard R stats package.

While glm2 should converge whenever glm converges, it may take a different path to convergence. This will occur whenever a convergent $\mathrm{glm}$ sequence has an iteration where the deviance increased. The fact that $g \mathrm{~lm} 2$ may take a different path to convergence should be inconsequential in practice, particularly as it will be a more stable monotone path.

Although we have found cases in which glm has convergence problems, in other contexts it copes very well with numerical difficulties. The data set from which the bootstrap replications were generated is an example. Using an identity link Poisson model, the existing step-halving in $\mathrm{glm}$ allows it to converge to the non-stationary MLE $\hat{\theta}=(0.578,-0.626,0.048,0.484)$. Since the fitted value for the observed covariate pattern $\left(x_{i 1}, x_{i 2}, x_{i 3}\right)=(1,1,0)$ is $0.578-0.626+0.048=0$, and all other fitted values are positive, the estimate produced is on the boundary of the parameter space. Not all GLM software would be able to converge to a non-stationary boundary point such as this.

Finally, we end with an observation on predicted values when using a non-standard link function. When glm (or glm2) converges with a link function that does not respect the natural parameter restrictions of the error distribution, such as in the example here, the predicted values will meet these restrictions for all observed covariate combinations. However, predicted values for other covariate combinations may not meet these restrictions. This is true not only for extreme covariate values outside the observed ranges, but also for unobserved combinations that are within the observed ranges. For example, the values $x_{i 1}=1, x_{i 2}=0$ and $x_{i 3}=0$ are all observed in the above data set, but the combination $\left(x_{i 1}, x_{i 2}, x_{i 3}\right)=(1,0,0)$ was not observed, and has a negative predicted value of $0.578-0.626=-0.048$. Although not available in $\mathrm{glm}$ or $\mathrm{glm} 2$, in principle it is possible to use a smaller parameter space that only has valid predicted values for covariate combinations that are within the observed ranges of the individual covariates. For the above data set this leads to the estimate $\hat{\theta}=(0.640,-0.640,0.000,0.476)$ (Marschner, 2010), which is slightly different to the one produced by $g \mathrm{~lm}$ and has a valid predicted value for the combination $(1,0,0)$. A discussion of the relative merits of these parameter spaces is not attempted here, but it is important to understand which one is being used when one fits a GLM with a non-standard link.

\section{Bibliography}

A. Agresti. An Introduction to Categorical Data Analysis. Wiley, Hoboken, USA, second edition, 2007.

K. Lange. Numerical Analysis for Statisticians. Springer, New York, USA, second edition, 2010.

I. C. Marschner. Stable computation of maximum likelihood estimates in identity link Poisson regression. Journal of Computational and Graphical Statistics, 19 (3):666-683, 2010.

I. C. Marschner. glm2: Fitting generalized linear models. 2011. URL http: / / CRAN.R-project.org/ package $=g l \mathrm{~m} 2$. $\mathrm{R}$ package version 1.0.

Ian Marschner

Department of Statistics

Macquarie University

NSW 2109

Australia

ian.marschner@mq. edu. au 